\title{
Recent Advances in Flame Tomography ${ }^{*}$
}

\author{
YAN Yong (间勇 $)^{1,2, * *}$, QIU Tian (邱天 $)^{1}$, LU Gang (卢钢 $)^{1,2}$, M. M. Hossain ${ }^{1}$, G. Gilabert ${ }^{1}$ and \\ LIU Shi (刘石) ${ }^{2}$ \\ ${ }^{1}$ School of Engineering and Digital Arts, University of Kent, Canterbury, Kent CT2 7NT, UK \\ 2 State Key Laboratory of Alternate Electrical Power System with Renewable Energy Sources, North China Electric \\ Power University, Beijing 102206, China
}

\begin{abstract}
To reduce greenhouse gas emissions from fossil fuel fired power plants, a range of new combustion technologies are being developed or refined, including oxy-fuel combustion, co-firing biomass with coal and fluidized bed combustion. Flame characteristics under such combustion conditions are expected to be different from those in normal air fired combustion processes. Quantified flame characteristics such as temperature distribution, oscillation frequency, and ignition volume play an important part in the optimized design and operation of the environmentally friendly power generation systems. However, it is challenging to obtain such flame characteristics particularly through a three-dimensional and non-intrusive means. Various tomography methods have been proposed to visualize and characterize flames, including passive optical tomography, laser based tomography, and electrical tomography. This paper identifies the challenges in flame tomography and reviews existing techniques for the quantitative characterization of flames. Future trends in flame tomography for industrial applications are discussed.

Keywords power generation, fossil fuel, biomass, combustion, flame, tomography, imaging
\end{abstract}

\section{INTRODUCTION}

The power generation industry is undergoing significant changes for economic and environmental reasons. Although the proportion of renewable energy will continue to grow, fossil fuels remain to be the main source of energy in many countries in the years to come. Coal-fired power stations are burning an increasingly varied range of fuels and fuel blends, including sub-bituminous and lower volatile coals of varying composition and combustion properties, under tight economic and environmental constraints. Since existing coal-fired power stations are not designed to burn such a diverse range of fuels, the power generation industry has to overcome a range of technological problems such as unstable combustion processes, poor combustion efficiency, increased pollutant emissions and other operational issues. The recent trend in operating power plant in variable load in response to changes in electricity demand in some European countries has exacerbated the aforesaid problems. To meet the increasingly stringent standards on combustion efficiency and pollutant emissions and to maintain fuel rangeability and operation flexibility, advanced flame monitoring techniques have become highly desirable [1].

In order to reduce greenhouse gas emissions from combustion-based power generation new technologies are being developed. One of these is oxyfuel combustion, which is regarded as a near-zero emission technology adaptable to both new and existing fossil-fuel fired power plants. In an oxyfuel firing system, pulverized fuel is fired in the re-circulated flue gas mixed with pure oxygen to produce a high concentration of $\mathrm{CO}_{2}$ in the flue gas stream which can be sequestrated without costly $\mathrm{CO}_{2}$ separation [2]. However, switching from the conventional air-fuel to oxyfuel brings a number of technological challenges to combustion engineers and plant operators. In the oxyfuel combustion process the increased concentrations of $\mathrm{CO}_{2}$ and water vapour in the flue gas would substantially increase the emissivity of the furnace gas and thus increase the radiative heat transfer in the furnace. In addition, the different heat capacity and densities of the main gases, i.e., $\mathrm{N}_{2}$ and $\mathrm{CO}_{2}$, will change the mass flows and velocities of the primary and secondary flows, and thus burner aerodynamics, resulting in changes in fuel ignition properties, flame propagation, shape, and residence time [2]. There is currently a significant lack of understanding of oxyfuel flames, so advanced flame monitoring and visualization techniques will help achieve an in-depth understanding and subsequent optimization of oxyfuel combustion processes.

Another topical issue in the power generation industry is the efficient utilization of biomass as a renewable energy source. Since in the process of biomass growth $\mathrm{CO}_{2}$ is absorbed from the atmosphere and biomass combustion releases $\mathrm{CO}_{2}$ back to the atmosphere, biomass is regarded as $\mathrm{CO}_{2}$-neutral. Although biomass can be used to generate energy in different ways, direct combustion or co-firing with coal at existing power stations remains a practical option available to plant operators and is widely adopted in the power industry for reducing carbon emissions. Biomass has higher moisture content and volatile matter than coal. Biomass particles are often highly irregular

Received 2011-06-24, accepted 2012-01-03.

* Supported by the National Natural Science Foundation of China (50736002, 61072005), the 1000-Talent-Plan, Changjiang Scholars and Innovative Team Development Plan (IRT0952), and partly by Research Councils United Kingdom's Energy Programme (EP/G063214/1).

** To whom correspondence should be addressed. E-mail: y.yan@kent.ac.uk 
in shape and greater in size compared to coal. Such differences between biomass and coal result in unstable flames and complex flame characteristics in biomass combustion and biomass/coal co-firing. On-line continuous monitoring of biomass or biomass/coal flames in power plant furnaces is imperative to maintain constant optimization of combustion conditions, leading to more stable flames, increased thermal efficiency, and reduced slagging/fouling problems.

A conventional flame detector uses a single infrared or ultraviolet photodetector, which is limited to a single point detection of the flame. Digital imaging techniques are an extension of the conventional optical detection approach and are capable of providing two-dimensional (2-D) information of a flame field. A range of measurements can be derived from 2-D flame images when a side view of the flame is available [3]. A number of 2-D imaging systems have been developed for flame monitoring and characterization on laboratory scale furnaces [2, 4-9]. However, a flame is naturally a three-dimensional (3-D) flow field. 3-D information about a flame is desirable to fully reveal the physical and chemical characteristics of the flame. Such information provides important data for combustion engineers to achieve an in-depth understanding of flames and subsequent optimization of combustion processes. There have been some efforts to develop 3-D flame visualization systems in recent years. In particular, there have been activities in 3-D reconstruction and measurement of flame geometrical information $[10,11]$. The 3-D measurement of temperature distribution in a furnace has also been conducted using multiple cameras and pyrometric techniques $[12,13]$. With the advent of digital imaging devices and computed tomography techniques, it has become feasible to visualize and reconstruct flames three-dimensionally. Such 3-D tomographic data will assist scientists and engineers working in combustion and related fields to study internal structures and flame front movement as well as other physical and chemical characteristics of flames. This paper presents a short review of the current research activities in flame tomography and addresses the technical challenges and future trends in this particular area of research.

\section{CHALLENGES IN FLAME IMAGING AND CHARACTERIZATION}

Despite recent progress in developing flame tomography techniques a range of challenges remain to be resolved. These include the design and implementation of a practical hardware platform that generates a sufficient number of flame projections and hence an acceptable spatial resolution and high reconstruction quality. Meanwhile, the development of computational algorithms that are suitable and efficient for flame tomographic reconstruction is required. Furthermore, the system should be suitable for installation on a furnace and capable of enduring the harsh environment in the power industry (particularly wide ambient temperature fluctuations and presence of dust). In addition, the system should be able to produce the measurements of a range of flame parameters from the reconstructed flame models. Such measurements can be broadly subdivided into following interrelated groups:

(1) Geometric parameters: including overall volume, ignition volume, surface area, and circularity as well as solid angle and orientation with reference to the burner outlet $[10,14]$.

(2) Luminous parameters: including brightness, uniformity and colour components.

(3) Fluid-dynamic parameters: temperature distribution, soot concentration distribution, particle velocity profile, oscillation frequency, and free radical concentrations $[3,7]$.

Some of the parameters are more important than others, depending on the specific purpose of the measurement and the system installation arrangement. It must be pointed out that flame tomography systems can provide 3-D information that would otherwise be impossible to obtain using 2-D imaging systems. For instance, a stable flame requires a steady flame front at which the heat lost and heat release of the fuel are well balanced at the ignition temperature of the fuel. The ignition volume is a measure of the normalized volume encompassed between the burner outlet, the flame front and the solid angle, which gives integrated information of flame ignitability.

\section{CURRENT FLAME TOMOGRAPHY TECH- NIQUES}

Techniques for flame tomography can be broadly categorized into passive optical tomography, laser based tomography, and electrical tomography.

\subsection{Passive optical tomography}

Since a flame is normally a luminous and translucent object, it can be imaged directly using one or more cameras without the use of a light source. 3-D tomographic models can then be built based on the 2-D images obtained in conjunction with tomographic reconstruction algorithms.

\subsubsection{Single-camera systems}

Several single-camera based flame tomography systems have been reported. Brisley et al. [15] proposed a single-camera system, as shown schematically in Fig. 1. The optical assembly splits the light of flame into two beams, which pass through two narrow-band optical filters of different wavelengths. The two images reach the camera for the tomographic reconstruction of the flame. A fundamental assumption is made such that the flame exhibits a rotational symmetry about the burner axis, i.e., the flame appears to be identical when observed from different perspectives. This assumption allows grayscale sections of the flame to be reconstructed using only one projection 


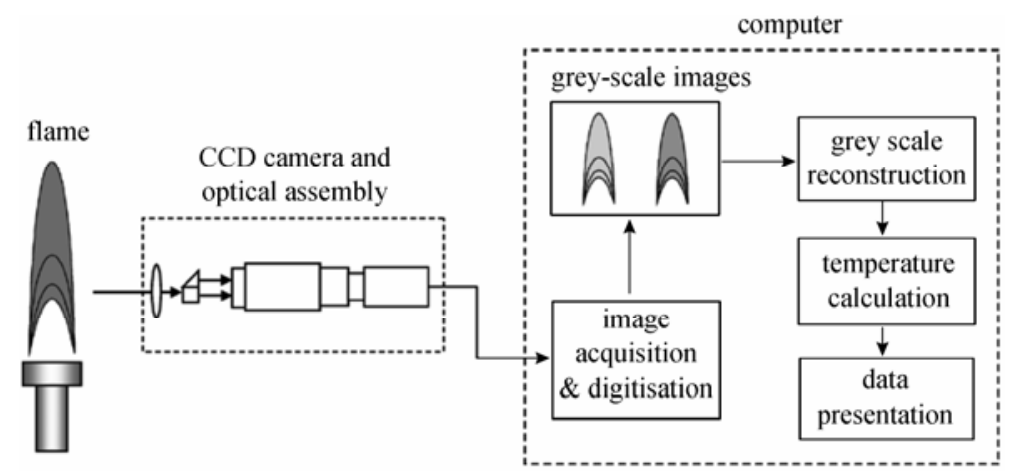

Figure 1 Single camera based 3-D flame temperature measurement system [15]

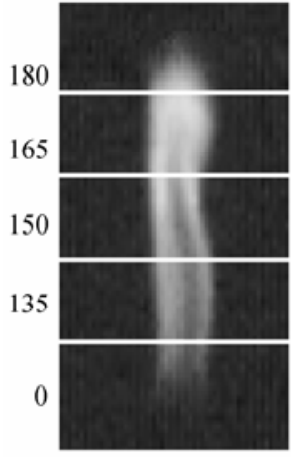

(a)

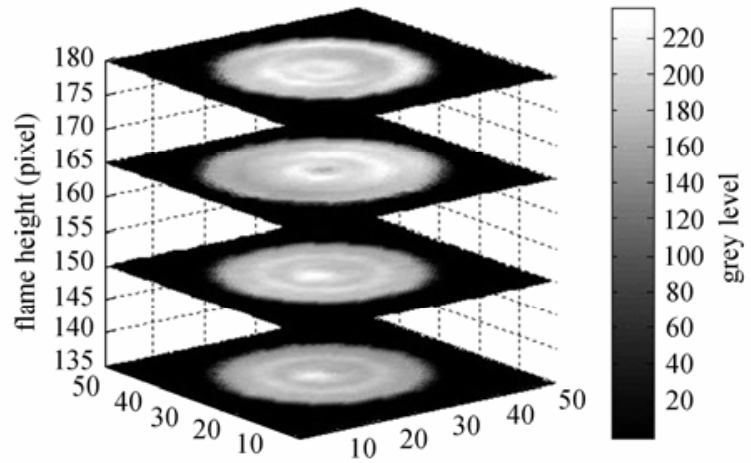

(b)

Figure 2 Reconstructed grayscale sections of a gas flame [15] (a) 2-D flame image, and (b) reconstructed 3-D flame sections

based on the FBP (Filtered Back Projection) algorithm. Fig. 2 shows an example of the reconstructed 3-D grayscale flame sections.

Huang et al. [16] developed a stereoscopic tomography technique to reconstruct the soot temperature and concentration distributions for an asymmetric diffusive flame. A CCD camera coupled with a stereoscopic adapter was used to capture flame images (Fig. 3). A computational algorithm working on the matrix decomposition-based least squares method was proposed to reconstruct the emission intensity distributions in the flame sections from the images obtained. The retrieved intensities were then used to infer local soot temperature and concentration. The proposed tomography system was capable of providing symmetric and asymmetric flame measurements. Fig. 4 shows the reconstructed temperature and soot concentration distributions of a gaseous flame.

Agard and Sedat [17] proposed an Optical Sectioning Tomography (OST) technique which uses a constrained de-convolution algorithm to obtain sharper and more detailed flame images. Zhou et al. [18] developed an OST system for the 3-D monitoring of a candle flame, in particular, the determination of flame temperature distribution. The flame is regarded as a combination of a number of layers of 2-D sections. Focusing on each layer of the flame, a set of images can be captured by a fixed CCD camera (Fig. 5). The original 2-D luminosity distribution of each section is

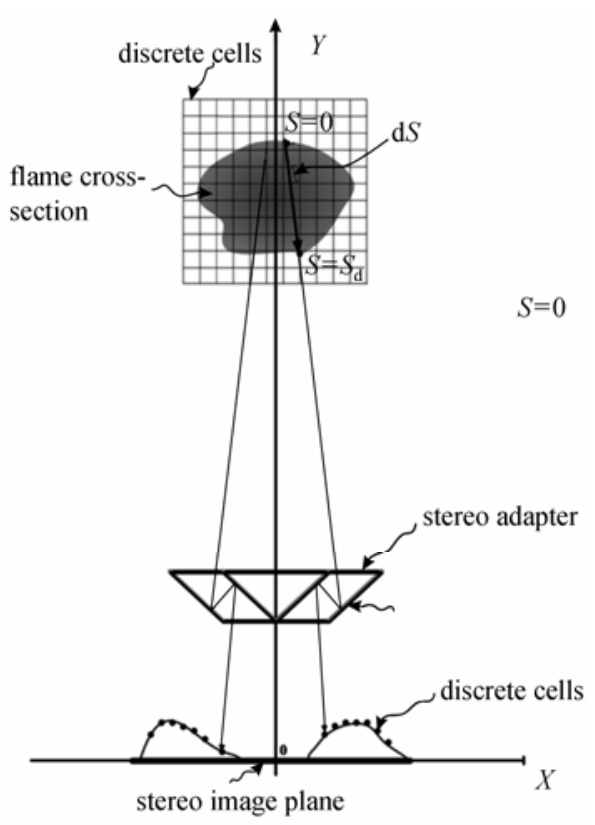

Figure 3 Stereoscopic image system [16]

retrieved through the supposition of section images to reconstruct the 3-D luminosity distribution of the flame. Fig. 6 shows 2-D images of a candle flame and the corresponding OST reconstruction results. 

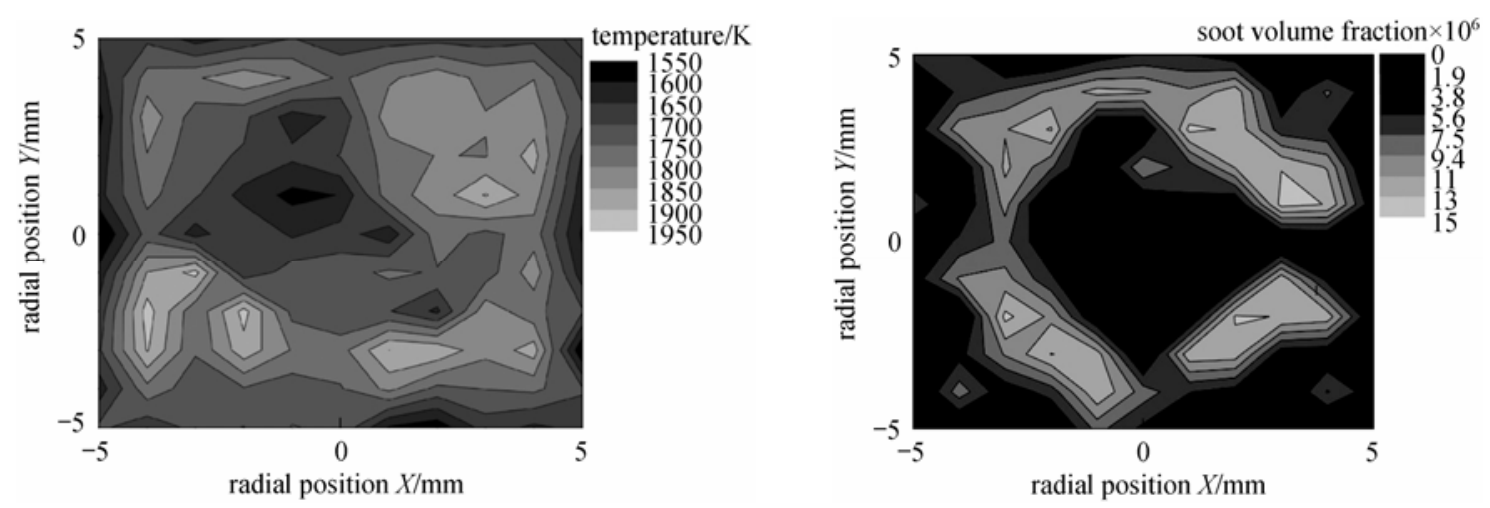

Figure 4 Reconstructed temperature and soot volume fraction distributions of a gaseous flame [16]

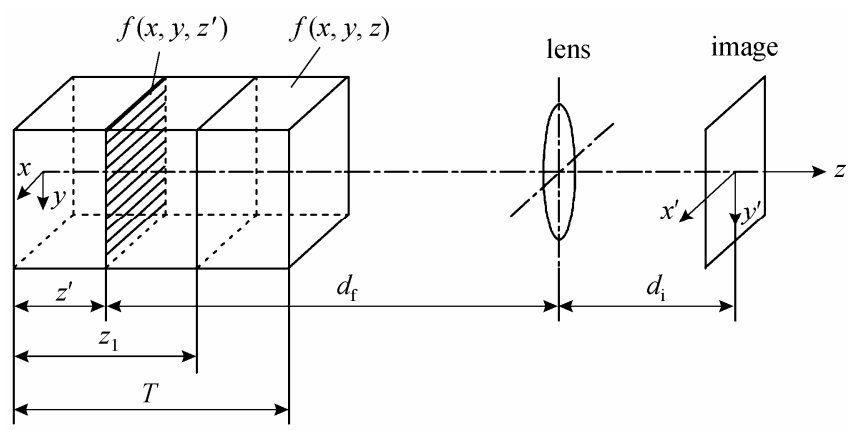

Figure 5 Principle of the Optical Sectioning Tomography [18]
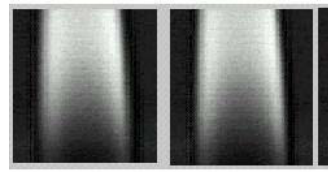

(a) Original gray-level images of different layers of the flame

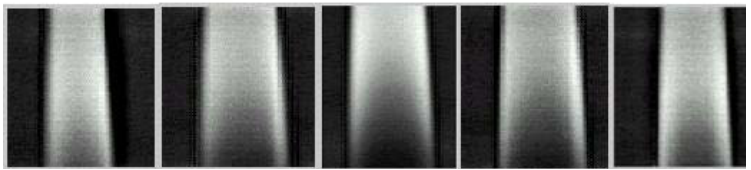

(b) Reconstructed gray-level sections of the flame

Figure 6 2-D images and OST reconstruction of a candle flame [18]

The single-camera systems are simple in structure, low in cost and can be installed on a practical furnace. It is also relatively straightforward to achieve 3-D reconstruction of the flame cross sections and to determine the 3-D temperature field. However, those techniques can only be used under strict conditions, i.e., the flame either has a high level of rotational symmetry or is very stable hence a limited temporal resolution resulting from adjusting the focal plane of the system to image different cross-sections is acceptable. Single-camera systems are unsuitable for monitoring unsteady, turbulent flames.

\subsubsection{Multi-camera systems}

Gilabert et al. [14] developed a three-camera system in which three identical colour CCD cameras together with optical transmission units were used to capture concurrently six equiangular 2-D images of a flame. The sensing arrangement is illustrated in Fig. 7. Several reconstruction algorithms, including FBP, ART (Algebraic Reconstruction Technique), LFBP (Logical FBP) and combined LFBP and ART, were compared.
The combined LFBP-ART algorithm was found to provide consistently better reconstruction results for the six projections system. The reconstruction results are of reasonably good quality - the internal structure of the flame can be clearly seen, as shown in Fig. 8. Despite the obvious improvement in the reconstruction of flames from multiple projections, more projections are still necessary for a higher spatial resolution. In addition, the system components are distributed around the flame, making it unsuitable for installation on an industrial furnace.

A tomography system using a bespoke multi-lens camera was used to obtain the instantaneous 3-D gray-scale distributions of a propane fuel-rich premixed turbulent flame [19]. The system (Fig. 9) is equipped with 40 special camera lenses (focal length $f=30 \mathrm{~mm}, F$ number $=3.0)$ arranged on a semi-circle on one side of the burner. A black-and-white panchromatic negative film (Fuji, Neopan 400) is loaded along the circumference behind the lenses and developed after the flame images are taken. The developed negative film is then digitized to obtain the digital 


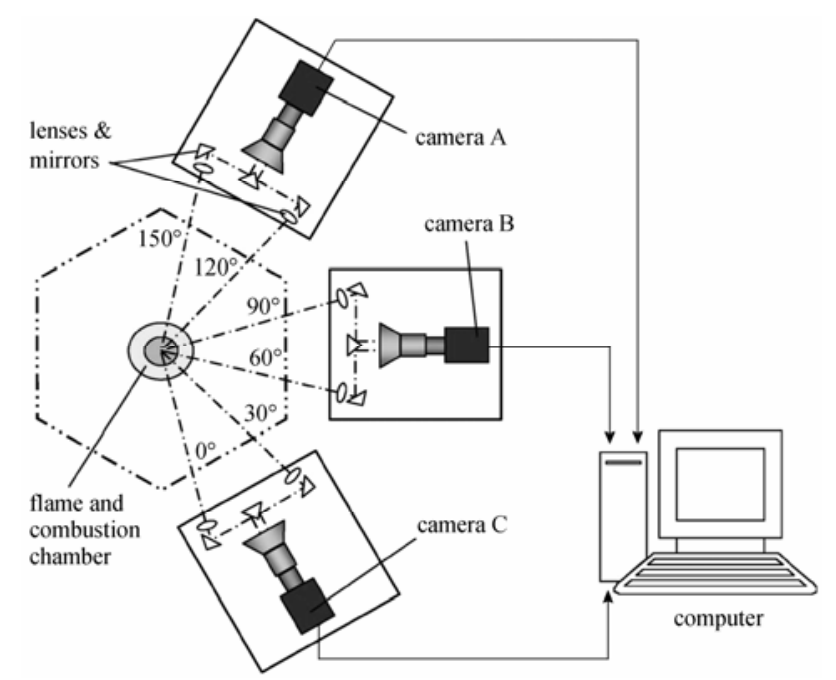

Figure 7 Three-camera six-projection system [14]

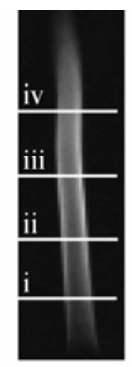

$0^{\circ}$ -D image

(a) Reconstructed cross-sections

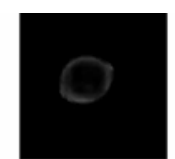

Section i

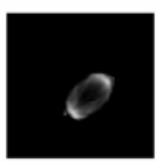

Section iii

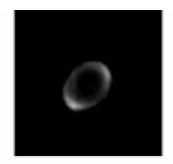

Section ii

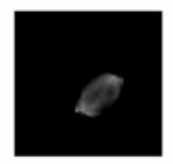

Section iv

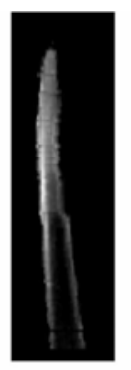

Section v

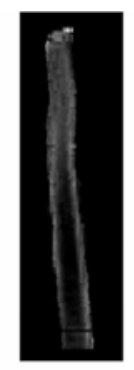

Section vi Section vii

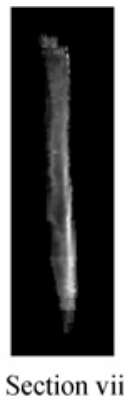

(b) Reconstructed longitudinal-sections

Figure 8 Luminosity reconstruction of a gaseous flame from six 2-D projections [14]

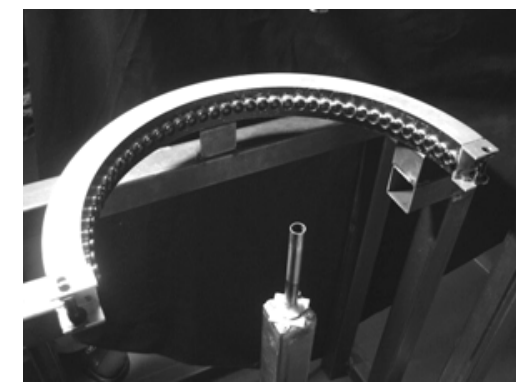

Figure 9 Forty-lens-camera tomography system [19]

projections of the flame. The camera has also a lineartracking mechanism to trace the flame stream up to $1.94 \mathrm{~m} \cdot \mathrm{s}^{-1}$, making it suitable for high-speed photography. Since the system uses a white-and-black film to capture 40 flame images at a time, the reconstructed 3 -D images are thus of a high resolution. Fig. 10 shows an example of the $3-\mathrm{D}$ reconstruction results. However, it is, because the system uses a film, the reconstruction process is complex and time-consuming, so the system is unsuitable for real-time flame monitoring. The complex arrangement of the imaging hardware makes the system installation on an industrial furnace difficult or impractical.

Multi-camera tomography systems are capable of acquiring multple flame projections around the burner and hence have a good spatial resolution. Higher the number of projections yields better reconstruction results. However, the increased number of cameras entails high system cost and complexity in system setup and installation. They are therefore restricted to laboratory use.

\subsection{Laser-based tomography}

Mohamad et al. [20] proposed a laser-based transmission tomography system to obtain the concentration profile of a gaseous flame. The system employs a single laser source with two orthogonal projections; each of them provides six parallel light beams reaching corresponding six photodiodes (Fig. 11). All the 12 light beams transmitted via optical fibers are from the same helium-neon light source. The ratio of the light intensity attenuated by the flame to that of the original light beam is used to reconstruct the cross-sectional image using an LBP (Linear Back Projection) algorithm. Fig. 12 illustrates the typical results of the reconstruction. 


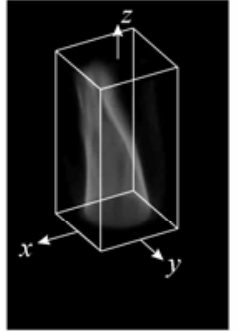

(a)

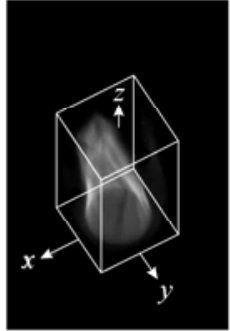

(b)

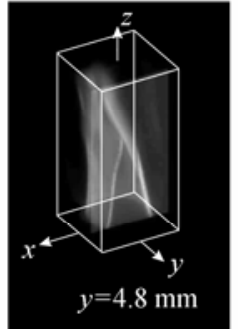

(c)

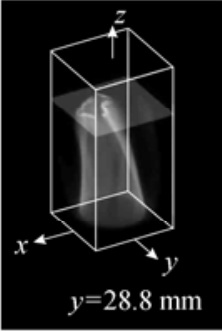

(d)

Figure 10 Reconstructed 3D images by forty-lens-camera system [19]

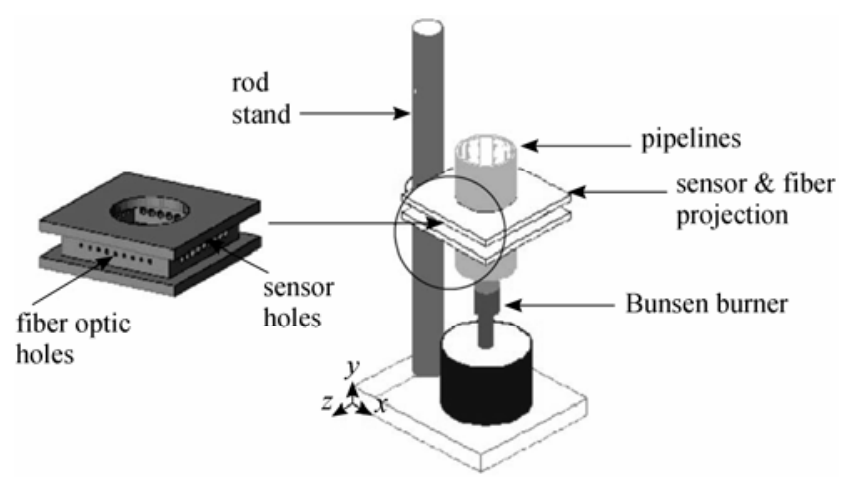

Figure 11 Setup of the laser based tomography system [20]
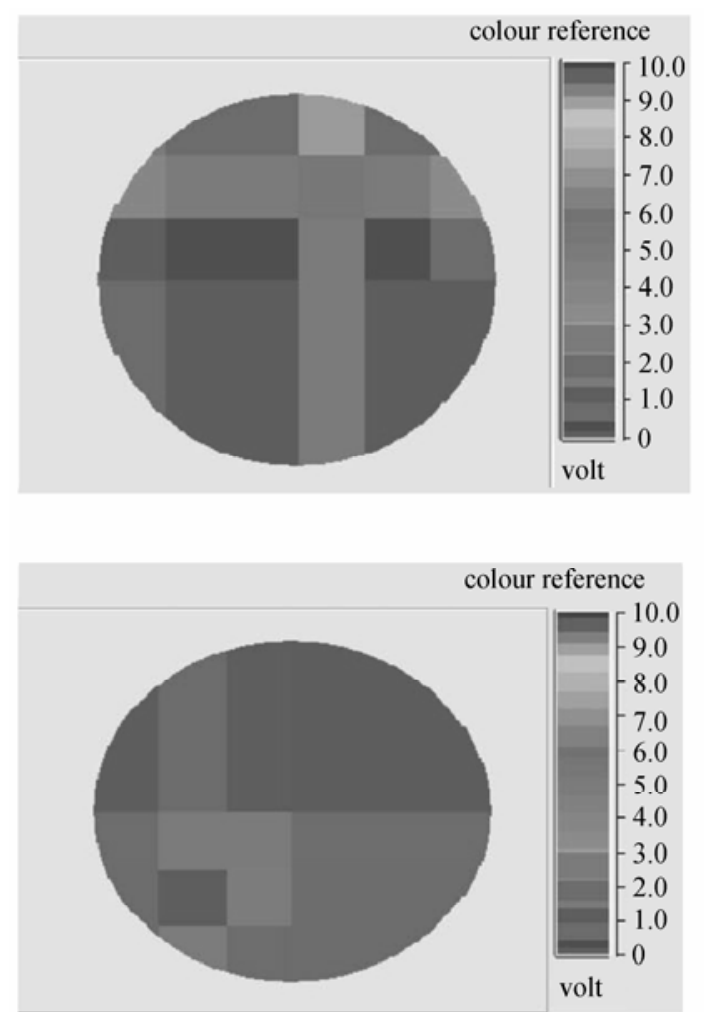
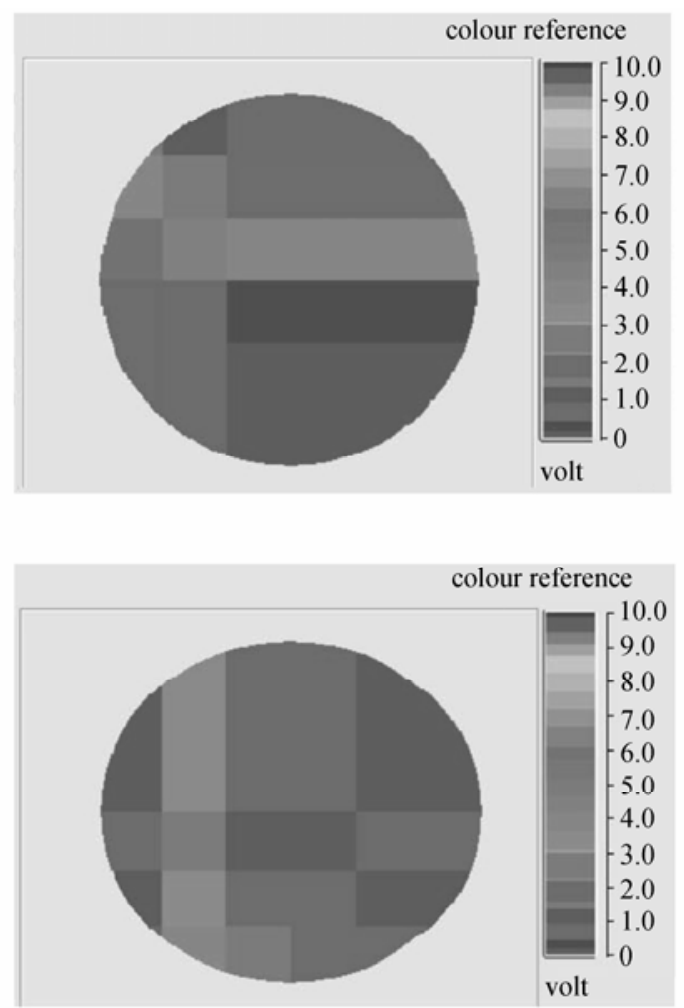

Figure 12 Reconstructed cross-sectional flame images [20]

These cross-sectional images may be used to analyze the effectiveness of different fuel additives or monitor the size, position and velocity of flame edge at a particular cross-sectional level. However, the spatial resolution of the system is poor and depends on the number of projection groups and the number of the laser beams. 


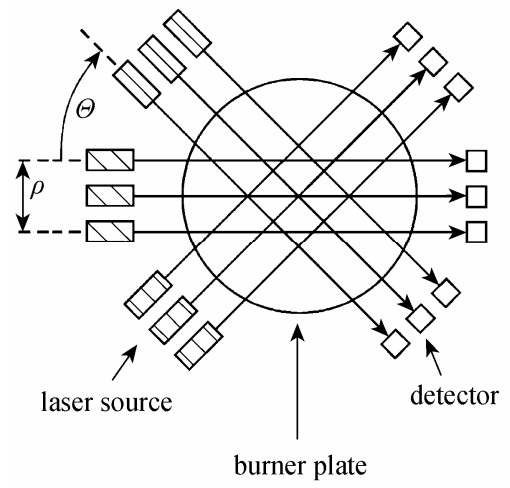

Figure 13 Laser and detector setup of a DFG-MIR system [21]

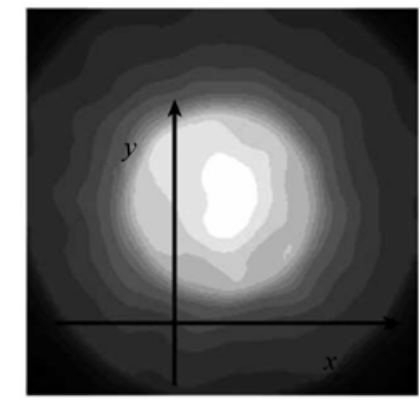

Figure 14 Reconstructed cross-sectional image by DFG-MIR system [21]

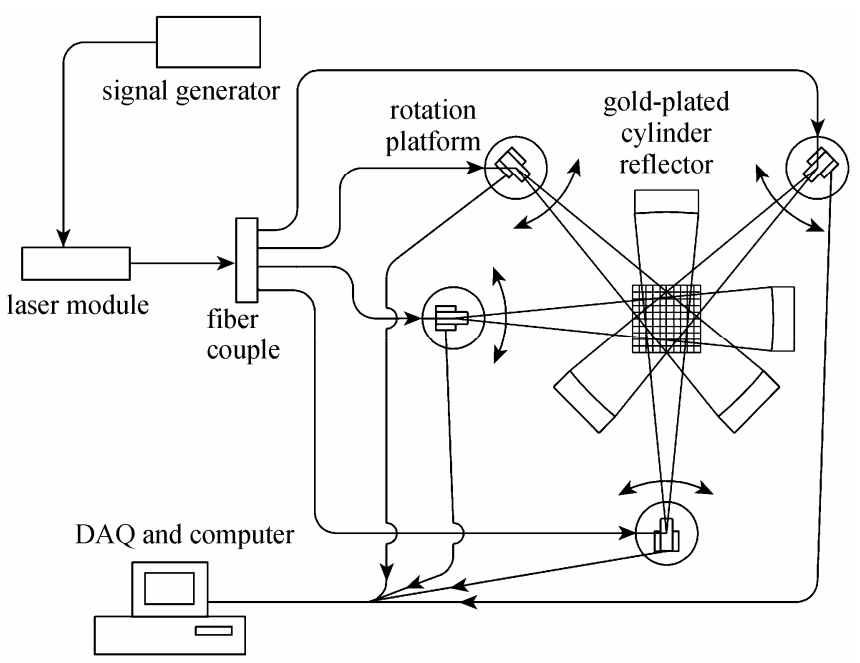

Figure 15 Schematic of the TDLAS tomography system [22]

Moreover, the hardware setup allows only one intersection of the flame to be obtained at one time.

Wondraczek et al. [21] developed an MIR (MidInfraRed) laser-based system for flame tomography. Two continuous wave, single-mode MIR diode lasers were used, which can be tuned from 680 to $682 \mathrm{~nm}$ and from 787 to $791 \mathrm{~nm}$, respectively. MIR lasers were used to avoid interference from the flame light. The system is of low spatial resolution because of the limited number of light transmitter-receiver pairs. To improve the resolution, additional projections were achieved by rotating the burner with an increment of $10^{\circ}$ and scanning of the flame in a 5-mm step (Fig. 13). In each layer $57(19 \times 3)$ data points were acquired around the burner axis. If five layers were scanned, a total of 285 data points were acquired for the tomographic reconstruction (Fig. 14). Like other laser based systems, it can only obtain one intersection reconstruction of the flame at a time.

Wang et al. [22] also developed a laser-based tomography system which combines the traditional line-of-sight TDLAS (Tunable Diode Laser Absorption Spectroscopy) with computerized tomography to determine the concentration distribution of specific chemical species for fluid mechanics and combustion applications. The system uses a fan-beam-based scheme with an incomplete set of projections using only four beams of a single laser source. A special optical design allows the spatial scan of each beam over an $11^{\circ}$ fan to be collected with a single detector using a curved retro-reflector (Fig. 15). This scheme reduces significantly the hardware and optical alignment costs of previous TDLAS tomographic imaging systems. The mole fraction and temperature distributions of $\mathrm{NH}_{3}$ mixture are reconstructed using the ART algorithm. Fig. 16 shows an example of the reconstruction results. The technique has the potential to be employed in investigations into the dynamic distribution of various gases for combustion diagnostics.

\subsection{Electrical tomography}

Flame tomography may be achieved through electrical means such as capacitance, impedance, and resistance. ECT (Electrical Capacitance Tomography), EIT (Electrical Impedance Tomography) and ERT (Electrical Resistance Tomography) have been attempted 

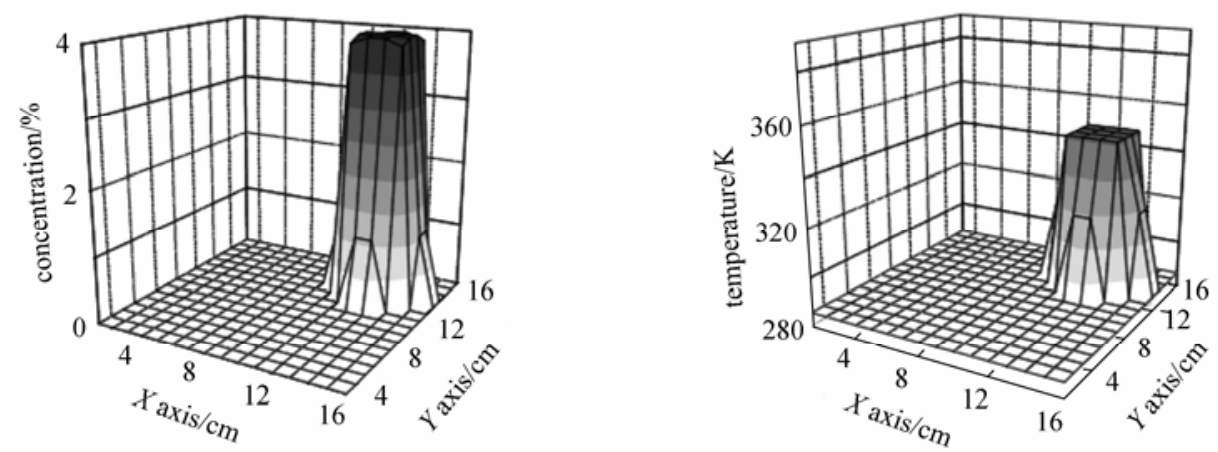

(a) Actual value
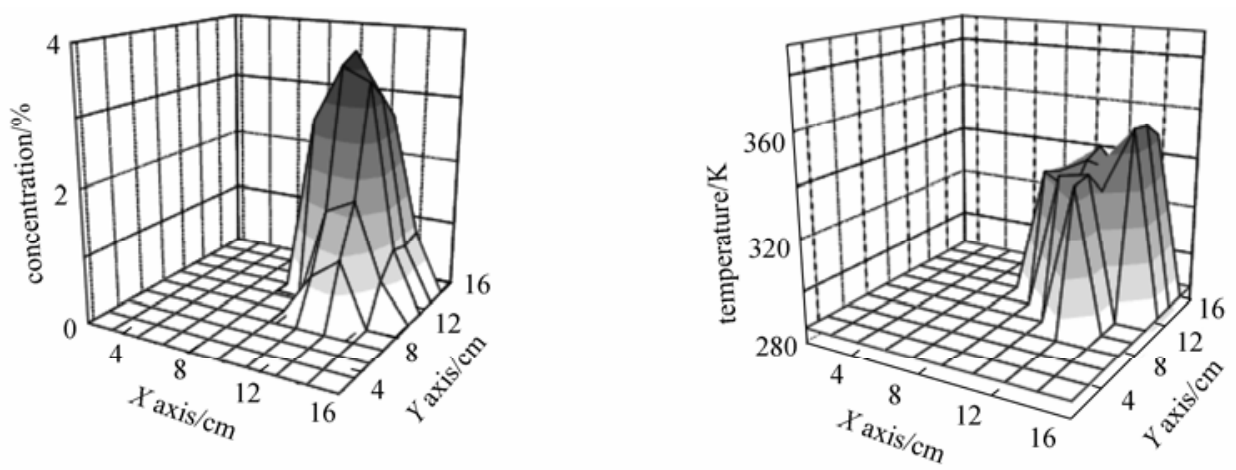

(b) Calculated value

Figure 16 Reconstructed concentration and temperature distributions of $\mathrm{NH}_{3}$ with the concentration of $4 \%$ in $\mathrm{N}_{2}$ and temperature of $324 \mathrm{~K}$ [22]

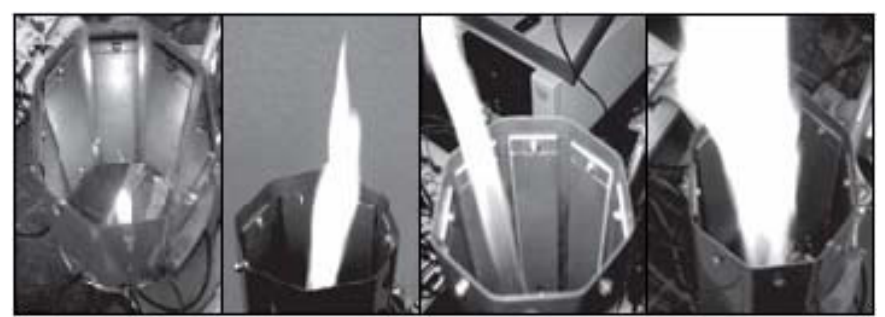

Figure 17 Small, larger, tilt and maximum fire in an ECT system [23]

for 3-D flame monitoring in recent years. Multiple projections of the flame are achieved by the use of 8 , 12 , or 16 electrodes around the burner.

The ECT measures the dielectric constant of a flame under investigation and is low cost and robust. A preliminary ECT system with eight electrodes for flame monitoring has been reported [23]. This system was used to study the distribution of a flame inside a chamber. Fig. 17 shows the experimental arrangement of the system. Data from the hardware system were used to reconstruct cross-sectional images using a conventional LBP algorithm (Fig. 18). The system is easy to set up and very low in cost and can be used to identify the approximate position of the flame in the chamber. However, as common with all ECT systems, its spatial resolution is poor both in the cross-sectional plane and in the vertical direction. This limitation makes the ECT fundamentally unsuitable for accurate 3-D flame tomography.

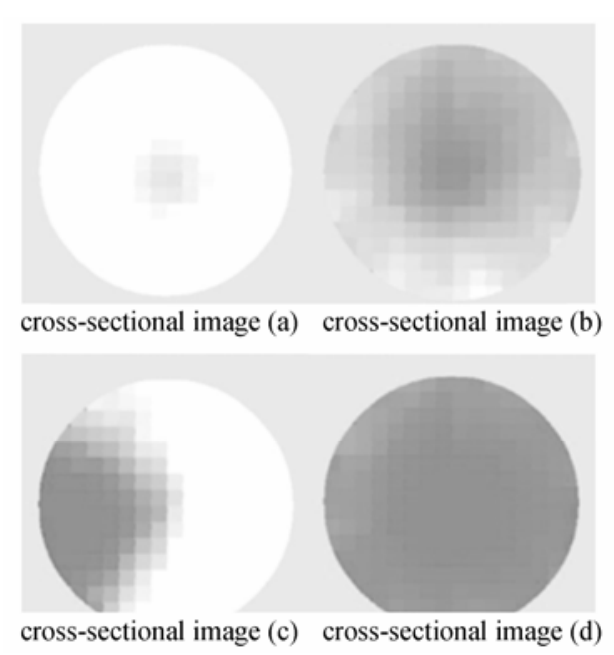

Figure 18 Reconstructed images through ECT [23] 


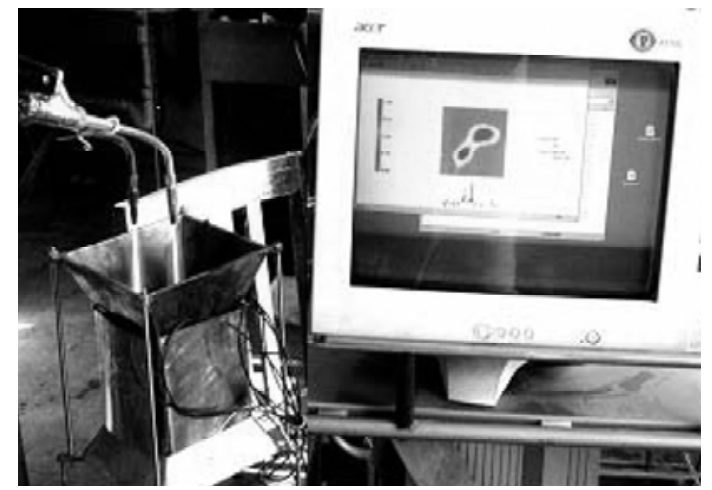

Figure 19 Demonstration of ECT imaging with porous block [24]

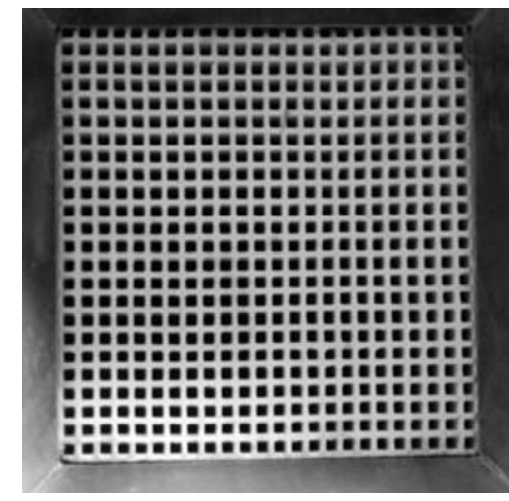

Figure 20 Porous block [24]
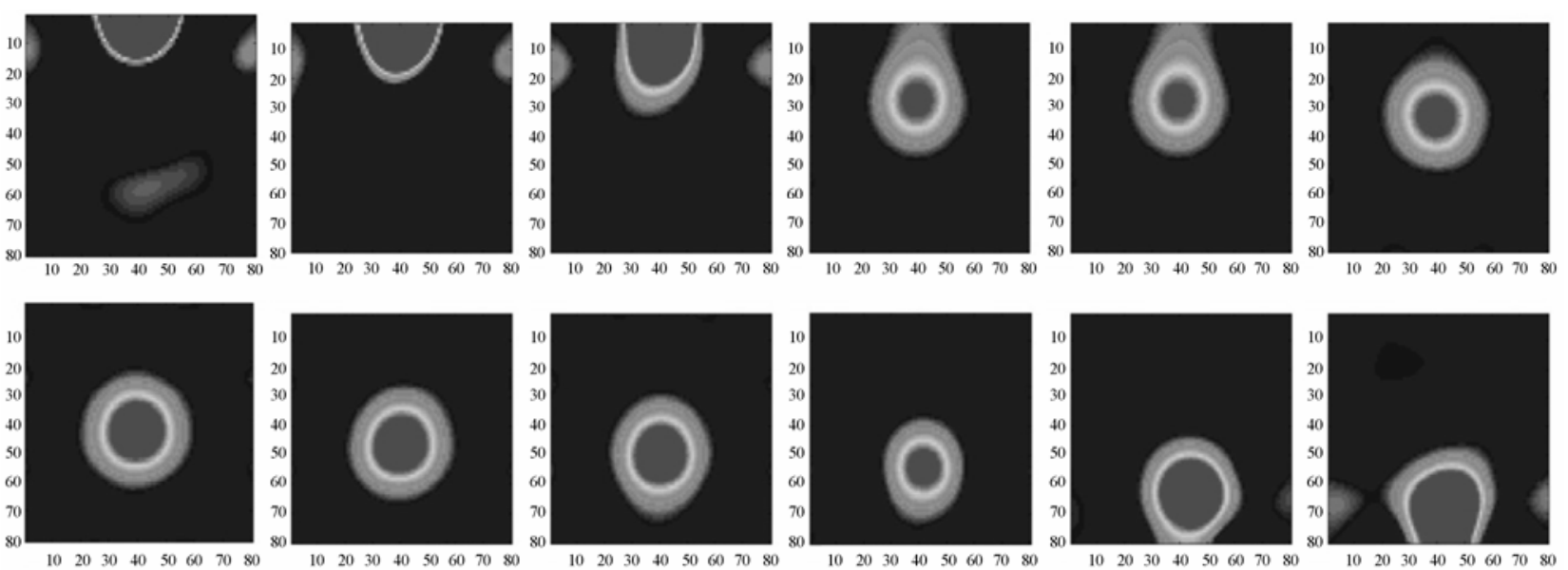

Figure 21 Reconstructed images through ECT imaging with porous block [24]

Liu et al. [24] also studied the feasibility of tomographic monitoring of flames in porous media using the ECT technique (Fig. 19). A ceramic block with a grid of straight-through holes (Fig. 20) was chosen as the porous medium. The holes are $2 \mathrm{~mm} \times 2 \mathrm{~mm}$ in size with 1 $\mathrm{mm}$ separation. The cross section of the block is 100 $\mathrm{mm} \times 100 \mathrm{~mm}$ and the height is $120 \mathrm{~mm}$. On its periphery of the block 12 electrodes were fixed as the ECT sensors. The relationship between the flame ionization and relative permittivity was established as the basis for ECT imaging of flames. The shape, size and motion of the flames in the porous block were monitored. The images were found to correspond to the variations of the combustion intensity (Fig. 21). Since many sensors are distributed in the porous block, this method can identify flame crossing-sections, while the porous block should be delicately designed to avoid the negative impact on the combustion process due to contact between the flame and the walls of the block.

EIT can be used to determine the electrical conductivity of a medium by making voltage and current measurements. ERT is closely related to EIT, and mathematically identical to EIT. In EIT the change of impedance is due to the type of medium under investigation. To measure resistivity or impedance, a current must flow through the medium and the corresponding voltages are measured. EIT techniques were applied to flame monitoring in 1980s and 1990s [25, 26]. There is no further report on the use of such techniques in recent years.

\section{FUTURE TRENDS}

Although there have been various advances in flame tomography using a range of sensing techniques as reviewed above, there are still substantial development work to be undertaken. None of the proposed techniques is capable of providing the identified 3-D flame parameters as described in Section 2. Many proposed systems are still at a laboratory stage. Some of the techniques are not suitable for the 3-D imaging of turbulent, dynamic flames due to their fundamental limitations in sensing principles. The following trends in future development are expected.

\subsection{Multi-projection tomography systems}

To achieve a reasonably good spatial resolution 
in flame tomography optical imaging techniques offer a better option than those based on electrical tomography. For the tomography point of view, the more optical projections of the flame, the better the reconstruction quality. However, a tomography system, which can generate a high number of flame projections or has a scanning part, is difficult to install on an industrial furnace. The challenge here is how to strike a balance between the numbers of projections required for high quality reconstruction results and the practicality for installation on industrial furnaces. The singlecamera systems are simplest in terms of hardware implementation and installation and can produce 3-D flame results in some cases, but they are unsuitable for applications under most conditions of practical furnaces where the flame is dynamic and irregular. The OST would be advantageous in several ways if the focal plane of the camera could be adjusted swiftly or a dedicated optical device is available for this purpose. The existing multiple camera systems that generate three or six projections, either with or without an external illumination source, performs better than the single-camera systems, but the reconstruction quality is still not satisfactory. The systems with a rotating or scanning mechanism, though producing a high number of projections and hence better reconstruction quality, have the same limitation as the single-camera systems. It is expected that flame tomography systems with an adequately higher number of projections (perhaps not as many as 40) will emerge in the near future. High performance digital imaging devices are becoming increasingly affordable and compact and the use of such multiple devices will become feasible. Alternatively, optical components such as fibres combined with miniature lenses may be used to generate more projections rather than using many cameras, which would result in high capital cost and installation complexity. Although the flame images have a slightly lower spatial resolution in such an approach and it may require more software work, it is practical in view of simplified system installation and affordable capital cost.

\subsection{Reconstruction algorithms}

Traditional tomography relies on interrogation (absorbency) data collected from multiple projections with the support of an external light source or beam that defines the interrogated slice of the object. In the case flame projections are acquired without the use of an external light or radiation source, the reconstruction algorithms should interpret the flame luminance data rather than the conventional absorbency data. It is not clear whether the classic algorithms such as FBP and ART are best for the passive optical flame tomography. For this very reason, reconstruction algorithms that are suitable for flame tomography should be developed. Prior knowledge of flames and computational flame modelling results should be combined with reconstruction algorithms to reduce the requirement of the higher number of projections. Almost all tomography algorithms take a huge amount of computation time to rebuild 3-D images. Efficient 3-D reconstruction algorithms should be sought to enhance the performance of flame tomography systems. Embedded image pre-processing and reconstruction architectures may be considered in future development.

\subsection{Measurement of 3D flame parameters}

There will be further development in tomography based measurement of 3-D flame parameters. In addition to 3-D flame temperature distribution where multiple cameras may be installed, additional information about the flame will be extracted from the reconstructed 3-D flame data. This additional information may include flame front movement, ignition patterns, oscillation frequency distribution, soot concentration distribution, concentrations of free radicals, and fuel/air balance in the presence of multiple burners. The various methods as outlined in Section 3 can be used to extract and determine the 3-D flame parameters. The methods may be enhanced by incorporating dedicated image processing algorithms [27, 28]. Flame tomography results will provide ample data for the validation of CFD models of the flames and furnaces, leading to optimized design and operation of coal fired plant firing a diverse range of fuels under variable load conditions.

\subsection{Practical implementation}

There are still no fully proven flame tomography systems that are in full operation on a laboratory test rig or on an industrial furnace. Cost-effective practical flame tomography systems that are designed for advanced combustion diagnosis are expected to emerge in the near future. Meanwhile, dedicated systems for advanced flame monitoring and visualization may be developed, too. Such systems may be relatively complex and expensive, but should be capable of providing high resolution, quantitative 3-D flame data that cannot be provided through other means. More specialized systems such as synchrotron X-ray tomography may also be expected.

\section{CONCLUSIONS}

This paper has described the requirements and challenges in flame tomography systems for applications in the power generation industry and other engineering sectors such as chemical engineering and steelmaking, appraised the existing techniques designed for flame tomography, and forecast the possible trends in future development. It is certain that a 3-D flame imaging system will help combustion engineers not only for the in-depth understanding of conventional and modern combustion processes but also for 
the on-line continuous monitoring and optimization of flames and burner conditions. This review of current state-of-the-art in the field has indicated that the some progress has been made in flame tomography in recent years, however, there is still a long way to go before we can see fully operational systems in industry. It is believed that substantial further work is required in developing practically useful flame tomography systems, both in hardware platforms and in reconstruction and characterization algorithms.

\section{REFERENCES}

1 Roddy, D., Advanced Power Plant Materials, Design and Technology, Woodhead Publishing (2010).

2 Smart, J., Lu, G., Yan, Y., Riley, G., "Characterisation of an oxy-coal flame through digital imaging”, Combus. Flame, 157, 1132-1139 (2010).

3 Lu, G., Yan, Y., Colechin, M., "A digital imaging based multi-functional flame monitoring system”, IEEE Trans. Instrum. Meas., 53, 1152-1158 (2004).

4 Yan, Y., Lu, G., Colechin, M., "Monitoring and characterisation of pulverised coal flames using digital imaging techniques", Fuel, 81 (5), 647-656 (2002).

5 Lu, G., Yan, Y., Cornwell, S., Whitehouse, M., Riley, G., "Impact of co-firing coal and biomass on flame characteristics and stability", Fuel, 87 (7), 1133-1140 (2008).

6 Molcan, P., Lu, G., Bris, T.L., Yan, Y., Taupin, B., Caillat, S., "Characterisation of biomass and coal co-firing on a 3 MWth combustion test facility using flame imaging and gas/ash sampling techniques", Fuel, 88, 2328-2334 (2009).

7 Krabicka, J., Lu, G., Yan, Y., “A spectroscopic imaging system for flame radical profiling”, In: IEEE Proceedings of International Instrumentation and Measurement Technology Conference (I2MTC 2010), 1387-1391, Austin, USA (2010).

8 Lou, C., Zhou, H., "Deduction of the two-dimensional distribution of temperature in a cross section of a boiler furnace from images of flame radiation", Combust. Flame, 143, 97-105 (2005).

9 Wang, F., Wang, X., Ma, Z., Yan, J., Chi, Y., Wei, C., Ni, M, Cen, K., "The research on the estimation for the $\mathrm{NO}_{x}$ emissive concentration of the pulverized coal boiler by the flame image processing technique", Fuel, 81 (16), 2113-2120 (2002).

10 Bheemul, H.C., Lu, G., Yan, Y., "Three-dimensional visualization and quantitative characterization of gaseous flames", Meas. Sci. Technol., 13 (10), 1643-1650 (2002).

11 Bheemul, H.C., Lu, G., Yan, Y., "Digital imaging-based threedimensional characterization of flame front structures in a turbulent flame”, IEEE Trans. Instrum. Meas., 54 (3), 1073-1078 (2005).

12 Luo, Z., Zhou, H., "A combustion-monitoring system with 3-D temperature reconstruction based on flame-image processing technique", IEEE Trans. Instrum. Meas., 56, 1877-1882 (2007)

13 Wang, H., Huang, Z., Wang, D., Luo, Z., Sun, Y., Fang, Q., Lou, C.,
Zhou, H., "Measurements on flame temperature and its 3D distribution in a 660 MWe arch-fired coal combustion furnace by visible image processing and verification by using an infrared pyrometer", Meas. Sci. Technol., 20 (11), 4006-4017 (2009).

14 Gilabert, G., Lu, G., Yan, Y., "Three-dimensional tomographic reconstruction of the luminosity distribution of a combustion flame", IEEE Trans. Instrum. Meas., 56 (4), 1300-1306 (2007).

15 Brisley, P.B., Lu, G., Yan, Y., Cornwell, S., "Three dimensional temperature measurement of combustion flames using a single monochromatic CCD camera", IEEE Trans. Instrum. Meas., 54 (4), 1417-1421 (2005).

16 Huang, Q., Wang, F., Liu, D., Ma, Z., Yan, J., Chi, Y., Cen, K., “Reconstruction of soot temperature and volume fraction profiles of an asymmetric flame using stereoscopic tomography", Combust. Flame, 156, 565-573 (2009).

17 Agard, D.A., Sedat, J.W., "3-D architecture of a polytene nucleus", Nature, 302, 676-681 (1983).

18 Zhou, B., Wang, S., Xu, C., "3-D flame temperature reconstruction in optical sectioning tomography", In: Proceedings of IEEE IST 2009-International Workshop on Imaging Systems and Techniques, Shenzhen, China (2009).

19 Ishino, Y., Hirano, T., Hirano, M., Ohiwa, N., "Non-scanning 3d-ct visualizations of premixed flames with a 40-lens camera", In: Proceedings of the 6th Pacific Symposium on Flow Visualization and Image Processing, A2-2, 1-6 (2007).

20 Mohamad, E.J., Rahim, R.A., Ibrahim, S., Sulaiman, S., Manaf, M.S., "Flame imaging using laser-based transmission tomography", Sensors and Actuators, A127, 332-339 (2006).

21 Wondraczek, L., Khorsandi, A., Willer, U., Heide, G., Schade, W., Frischat, G.H., "Mid-infrared laser-tomographic imaging of carbon monoxide in laminar flames by difference frequency generation", Combust. Flame, 138, 30-39 (2004).

22 Wang, F., Cen, K.F., Li, N., Jeffries, J.B., Huang, Q.X., Yan, J.H., Chi, Y., "Two-dimensional tomography for gas concentration and temperature distributions based on tunable diode laser absorption spectroscopy”, Meas. Sci. Technol., 21, 045301, 1-10 (2010).

23 Rahim, R.A., Chan, K.S., Sallehudin, I., "Fire-flame imaging using electrical capacitance tomography", J. Technol., 45 (D), 135-152 (2006).

24 Liu, S., Chen, Q., Xiong, X., Zhang, Z., Lei, J., "Preliminary study on ECT imaging of flames in porous media", Meas. Sci. Technol., 19, 1-7 (2008).

25 Hertz, H.M., "Experimental determination of 2-D flame temperature fields by interferometric tomography", Opt. Commun., 54 (3), 131-136 (1985)

26 Waterfall, R.C., He, R., White, N.B., Beck, C.M., "Combustion imaging from electrical impedance measurements", Meas. Sci. Technol., 7, 369-374 (1996).

27 Qiu, T., Yan, Y., Lu, G., “An auto-adaptive edge detection algorithm for flame and fire image processing”, IEEE Trans. Instrum. Meas., 99, 1-8 (2011).

28 Qiu, T., Yan, Y., Lu, G., "Watershed transformation based Identification of the combustion region in an oxy-coal flame image", In: Proceedings of the 6 th International Conference on Image and Graphics, 182-186, Hefei, China (2011). 